\title{
32. Zu neuen Ufern: \\ Die Entdeckung der jüdischen Geschichte
}

W olfgang Beck ist kein Mann großer Worte. Anderes ist ihm wichtiger als lange An-
Ich bin selbstverständlich sehr dafür, dass wir uns um Friedländers Buch bemühen.

Wolfgang Beck an Ernst-Peter Wieckenberg, 13. Juli 1992'

sprachen. Am 25. Februar 1991 aber hielt er vor großem Publikum eine Rede, die ihm persönlich am Herzen lag. Damals eröffnete er in der Aula der Ludwig-Maximilians-Universität die siebenteilige Vorlesungsreihe «Die Juden in der europäischen Geschichte». Nur ein paar Meter entfernt von jenem Lichthof, in dem Sophie und Hans Scholl mit den Flugblättern der Weißen Rose zum Widerstand gegen das Naziregime aufgerufen hatten, erinnerte der Verleger an die Bedeutung der judaistischen Forschungen, die in Deutschland bis 1933 betrieben worden waren und im «Dritten Reich» ihr abruptes Ende gefunden hatten. Deshalb hatte der Verlag fast 6o Jahre später namhafte jüdische Gelehrte eingeladen, um in München über wesentliche Etappen und Themen der jüdischen Geschichte in Mitteleuropa zu sprechen. Zugleich bekannte sich der Verleger einen guten Monat nach dem Ausbruch des Zweiten Golfkriegs und zwei Tage nach dem Beginn der alliierten Bodenoffensive öffentlich zu Israel: «Die Deutschen» seien «aufgrund dessen, was unter Hitler geschehen ist, zu kritischer Solidarität, zu besonderer Achtsamkeit, Sensibilität, Urteilsfähigkeit und Verantwortung den Juden und dem jüdischen Staat Israel gegenüber aufgerufen, ja verpflichtet.»² Die Vorlesungsreihe war mehr als nur ein Verlagsprojekt, in dessen Zentrum die jüdische Geschichte stehen sollte. Die Erinnerung an den Holocaust ließ Wolfgang Beck die Überzeugung formulieren, die in weiten Teilen der deutschen Öffentlichkeit geteilt wurde, dass Deutschland in dieser Situation besonders eng an der Seite Israels stehen müsse. Die Vorlesungsreihe war für den Verleger zugleich aber auch eine persönliche moralische Verpflichtung, die nicht zuletzt aus dem Unrecht resultierte, an dem der Verlag im «Dritten Reich» beteiligt gewesen war. Deshalb war es Wolfgang Beck zweieinhalb Jahre zuvor, als C.H.Beck sein 225-jähriges Jubiläum beging, ein dringendes Anliegen gewesen, dieses Vorhaben zu lancieren. Bald schon konnten als wissenschaftlicher Beirat 
für die Vorlesungsreihe die beiden Münchner Historiker Christian Meier und Thomas Nipperdey gewonnen werden.

\section{Jüdische Geschichte bei C.H.Beck vor 1991}

Judaica gehören seit dem 19. Jahrhundert zum Programm des großen Universalverlags. Am Anfang waren es protestantische Theologen, die sich mit der hebräischen Sprache sowie der jüdischen Geschichte und Religion beschäftigten. Damals wurde die jüdische Überlieferung als Teil des christlichen Erbes wahrgenommen, wie gerade das Lebenswerk des Berliner Theologen und Judaisten Hermann L. Strack zeigt, dessen «Einleitung ins Alte Testament» seit den 1880er Jahren in mehreren Auflagen erschien. 1903 begann Strack seine «Clavis linguarum semiticarum» herauszugeben, die Theologen und Philologen in die Sprachen des Alten Orients einführen sollte. ${ }^{3}$ Er selbst eröffnete die Reihe mit einer hebräischen Grammatik mit Übungsbuch, von der insgesamt 15 Auflagen erschienen sind; die letzte von $195^{2}$ bearbeitete der Rostocker Alttestamentler Alfred Jepsen, dessen Untersuchung «Nabi. Soziologische Studien zur alttestamentlichen Literatur und Religionsgeschichte» 1934 ebenfalls in München veröffentlicht worden war. Stracks Werk und Biographie verdeutlichen die Ambivalenz der christlich motivierten Auseinandersetzung mit der Geschichte und Religion des Judentums. Strack förderte energisch ein vertieftes Verständnis der jüdischen Kultur und stritt mutig gegen die antisemitischen Hetzschriften seiner Zeitgenossen, aber er hielt immer an dem christlichen Missionsziel fest; zu diesem Zweck gab er seit 1885 die Zeitschrift «Nathanael. Für die Arbeit der evangelischen Kirche an Israel» heraus und leitete das «Institutum Judaicum» an der Berliner Universität. ${ }^{4}$ Strack wollte die Deutungshoheit auch über das nachbiblische Judentum in christlicher Hand wissen. ${ }^{5}$

Gleichfalls unter Stracks Namen erschien seit 1922 der bereits erwähnte «Kommentar zum Neuen Testament aus Talmud und Midrasch», der eigentlich das Lebenswerk des Pfarrers Paul Billerbeck war. Dieses grundlegende Werk, das immer wieder neu aufgelegt wurde, überzeugte auch nach dem Zweiten Weltkrieg einzelne Alttestamentler und Judaisten, zu C.H.Beck zu kommen. Unter ihnen war der bekannte Leipziger Alttestamentler Albrecht Alt, dessen «Kleine Schriften zur Geschichte des Volkes Israel» in drei Bänden zwischen 1953 und 1959 (2. bzw. 4. Aufl. 1968/78) veröffentlicht wurden. Doch zu einem Fachverlag der alttestamentlichen 
Wissenschaft wurde C.H.Beck nicht. Auf diesem Gebiet trat man nicht in Konkurrenz mit traditionsreichen Häusern wie etwa Mohr Siebeck in Tübingen, W. Kohlhammer in Stuttgart oder Vandenhoeck \& Ruprecht in Göttingen.

Die Judaistik im Beck'schen Verlagsprogramm lebte bis in die zweite Hälfte der 1970er Jahre hinein von der Neuauflage älterer Werke. Dann fand der Wiener Judaistik-Professor Günter Stemberger zum Verlag, der 1977 in den «Beck'schen Elementarbüchern» seine «Geschichte der jüdischen Literatur» veröffentlichte. Zwei Jahre später führte er ein in «Das klassische Judentum. Kultur und Geschichte der rabbinischen Zeit» (1979, Neubearbeitung 2009). 1982 erschien die von ihm völlig neu bearbeitete siebte Auflage der inzwischen klassischen «Einleitung in Talmud und Midrasch» von Hermann L. Strack (9. Aufl. 2011). Es folgten «Epochen der jüdischen Literatur, an ausgewählten Beispielen erläutert» (1982), «Der Talmud: Einführung, Texte, Erläuterungen» (1982, 4. Aufl. 20o8), «Die Juden und Christen im «Heiligen Land〉. Palästina unter Konstantin und Theodosius» (1987), «Midrasch. Vom Umgang der Rabbinen mit der Bibel. Einführung, Texte, Erläuterungen» (1989, Sonderausgabe 2002), «Die Juden. Ein historisches Lesebuch» (1990, 4. Aufl. 1995), «Jüdische Religion» (1995, 6. Aufl. 2009) und die «Einführung in die Judaistik» (2002). Mit Stembergers Publikationen wurde die Geschichte des frühen Judentums im Verlagsprogramm auf eine neue Grundlage gestellt.

Gleichzeitig weitete man aber auch den zeitlichen Fokus. Die dreibändige «Geschichte des jüdischen Volkes» (1978-80, 5. Aufl. in einem Band 2007), die der israelische Historiker Haim Hillel Ben-Sasson herausgegeben hatte und die ursprünglich auf Hebräisch und dann in englischer Übersetzung erschienen war, erstreckte sich von den Anfängen bis zur Gegenwart und setzte neue Akzente in der Erforschung der jüdischen Geschichte und Kultur. Ernst-Peter Wieckenberg hatte auf einer Reise nach Israel Ende der 1970er Jahre bereits Kontakte zu israelischen Verlagen und Instituten hergestellt, der Hinweis auf die dreibändige jüdische Geschichte kam jedoch von einem Münchner Verlagskollegen, Walter Kumpmann von dtv. ${ }^{6}$ Mit der Veröffentlichung dieses Werkes begann der Verlag, sich auf einem Gebiet zu bewegen, das ihm in Zukunft ein hohes Maß an nationaler und internationaler Visibilität sicherte. Parallel zu den Bemühungen, ein Programm zur jüdischen Geschichte aufzubauen, versuchte man die Historie auch anderer Kulturen und Religionen, überhaupt die Religionsgeschichte und -wissenschaft stärker im Programm zu verankern. Auf all diesen Gebieten konnte man zunächst noch keine berühmten 
Autoren gewinnen. So waren die Anfänge bescheiden, während später bedeutende Fachgelehrte wie Jan Assmann, Friedrich Wilhelm Graf und Saul Friedländer - um nur einige zu nennen - zu C.H.Beck stießen.

Der Verlag überwand in den siebziger und achtziger Jahren eine exklusive christliche und okzidentale Perspektive auf die jüdische Geschichte und nahm nicht mehr nur das Judentum im Altertum, sondern die gesamte jüdische Geschichte und Kultur in den Blick. Folgerichtig wurde die Verfolgung der Juden durch das nationalsozialistische Deutschland zu einem Programmschwerpunkt ausgebaut. Ernst-Peter Wieckenberg war durch seine Tätigkeit bei S. Fischer für dieses Thema sensibilisiert. 1981 diskutierte man auf einer der Killy-Konferenzen die Möglichkeiten, eine «Dokumentation über die Judenpolitik im «Dritten Reich» zu realisieren. ${ }^{7}$ Damals war die Auseinandersetzung mit der nationalsozialistischen Judenverfolgung nicht zuletzt durch die Ausstrahlung des Fernsehvierteilers «Holocaust - Die Geschichte der Familie Weiss» zu einem Medienereignis geworden. Die Serie gilt inzwischen als ein «Wendepunkt der medialen Erinnerungskultur». ${ }^{8}$ Historische Identitätsstiftung wurde zu einem weithin beachteten Thema des öffentlichen Diskurses und Gegenstand politischer Kontroversen. Aufsehen erregten in den nächsten Jahren Helmut Kohls Besuch mit Ronald Reagan auf dem Soldatenfriedhof in Bitburg, auf dem sich auch die Gräber von Angehörigen der Waffen-SS befinden, und Richard von Weizsäckers Rede zum 8. Mai, die er 1985 im Deutschen Bundestag hielt und die den «Tag der Niederlage» zu einem «Tag der Befreiung» machte. Das «Dritte Reich», der Zweite Weltkrieg und die Shoah fanden in der außerwissenschaftlichen Öffentlichkeit weithin Beachtung. Doch damals war «die Zahl der wissenschaftlichen Arbeiten von westdeutschen Historikern über den Judenmord [...] bemerkenswert gering». ${ }^{9}$ Bei C.H.Beck gab Wolfgang Benz, seinerzeit Mitarbeiter des Münchner Instituts für Zeitgeschichte, erst 1988, als sich das Pogrom vom November 1938 zum fünfzigsten Mal jährte, den Band «Die Juden in Deutschland 19331945" heraus. Die Zusammenarbeit mit Benz, der seit 1990 das Zentrum für Antisemitismusforschung an der TU Berlin leitete, dauerte an. Er veröffentlichte zahlreiche seiner Werke bei C.H.Beck, darunter die wichtigen Bücher «Der Holocaust» (1995, 7.Aufl. 2008), «Geschichte des Dritten Reiches» (2000) sowie - zusammen mit Barbara Distel und Angelika Königseder - die neun Bände des Grundlagenwerks «Der Ort des Terrors. Geschichte der nationalsozialistischen Konzentrationslager» (2005-09). 
Wolfgang Beck und Ernst-Peter Wieckenberg wollten indes nicht nur Bücher zur Judenverfolgung im Verlag herausbringen. Die gesamte deutsche, ja europäische Geschichte des Judentums sollte behandelt werden. Aber es fehlte an potentiellen Autoren. In Deutschland gab es keinen Lehrstuhl für jüdische Geschichte. Man musste sich im Ausland umschauen. Deshalb war die Vortragsreihe so konzipiert, dass «namhafte jüdische Gelehrte in die Bundesrepublik» eingeladen wurden. ${ }^{10}$ Doch wer hatte die Idee zu diesem Projekt? Der kulturwissenschaftliche Verlag sollte, so schlug Eva von Freeden vor, anlässlich des Verlagsjubiläums etwas «mit Nutzen für die Geschichtswissenschaft» tun. Ernst-Peter Wieckenberg sprach mit Thomas Nipperdey, der anregte, eine Vorlesungsreihe zu stiften, «die vorsähe, dass einmal im Jahr über mehrere Jahre hinweg eine wie auch immer zu nennende Vorlesung» an der Münchner Universität gehalten würde. Das Projekt wurde dann im Sommer 1988 gemeinsam mit Christian Meier, der damals Präsident des Historikerverbands war, besprochen. ${ }^{11}$ Der Althistoriker hatte zwei Jahre zuvor in weithin beachteten Beiträgen zur «deutschen Geschichtserinnerung» und zum Historikerstreit Stellung genommen; und er hatte nicht nur im Oktober 1986 auf dem Historikertag in Trier den Eröffnungsvortrag gehalten, sondern zuvor bereits in Tel Aviv über das schwierige Verhältnis der Deutschen zu ihrer Geschichte gesprochen. ${ }^{12}$ Damit stand das Thema der Vorlesungsreihe fest: «Deutsche und Juden in der Geschichte».

Lange und intensiv diskutierten Ernst-Peter Wieckenberg und die beiden Historiker in der Folge über mögliche Redner und Themen. Listen wurden erstellt, Namen ergänzt, andere verworfen; man wechselte Briefe, traf sich zum Gespräch, telefonierte. Wieckenberg fragte Monika Richarz um Rat, die damals die Leitung der Germania Judaica, der Kölner Bibliothek zur Geschichte des deutschen Judentums, innehatte, und Shulamit Volkov, die 1990 zehn Essays über «Jüdisches Leben und Antisemitismus im 19. und 2o. Jahrhundert» publizierte. Zudem kontaktierte Wieckenberg Fred Grubel, den Vizepräsidenten des Leo Baeck Instituts. ${ }^{13}$ Der Cheflektor verfügte über gute Beziehungen nach New York: 1989 erschien als Veröffentlichung des Leo Baeck Instituts bei C.H.Beck die von Itta Shedletzky herausgegebene Korrespondenz zwischen Betty und Gershom Sholem: «Mutter und Sohn im Briefwechsel 1917-1946». Schließlich entschied man sich für sieben «international bekannte Gelehrte», ${ }^{14}$ die im August 1990 kontaktiert wurden, um sie zu Vorlesungen zwischen Februar 1991 und 
Juli 1992 nach München einzuladen. Ein breites Publikum sollte adressiert werden, und deshalb war es dem Verlag wichtig, dass die Vorlesungen auf Deutsch gehalten würden. Für die Übersetzung bot man Hilfe an. Inzwischen war der Titel der Reihe in «Die Juden in der europäischen Geschichte» geändert worden, um die Perspektive zu weiten und eine größere Auswahl unter den potentiellen Rednern zu haben. ${ }^{15}$

Am 25. Januar 1991 eröffnete der einzige deutsche Wissenschaftler in diesem Kreis, der Stuttgarter Historiker Eberhard Jäckel, die Reihe; er war anstelle von Monika Richarz angefragt worden, die abgesagt hatte. Jäckel sprach über den «Mord an den europäischen Juden und die Geschichte». Es folgten am 27. Mai 1991 Amos Funkenstein aus Tel Aviv, dessen Vortragsthema «Juden, Christen und Muslime. Religiöse Polemik im Mittelalter» lautete; am 15. Juli 1991 referierte David Sorkin aus Oxford über «Juden und Aufklärung: Religiöse Quellen der Toleranz»; am 4. November 1991 kam Michael A. Meyer aus Cincinnati, der in der Betrachtung der «jüdischen Reformbewegung in Deutschland in jüdischer und christlicher Sicht» eine Antwort auf die Frage «Soll und kann eine «antiquierte Religion modern werden?» suchte; am 10. Februar 1992 thematisierte Shulamit Volkov aus Tel Aviv die «Juden im Zeitalter der Emanzipation. Einheit und Vielfalt», und am 18. Mai $199^{2}$ sprach Jehuda Reinharz von der Brandeis University in Massachusetts über «Jüdische Identität in Zentraleuropa vor dem Zweiten Weltkrieg». Der letzte Redner war Saul Friedländer. Der Professor an der Hebrew University in Jerusalem, der zugleich den Lehrstuhl für Geschichte des Holocausts an der University of California in Los Angeles innehatte, verglich am 6. Juli 1992 «Trauma, Erinnerung und Übertragung in der historischen Darstellung des Nationalsozialismnus und des Holocaust». Damit waren herausragende Historiker und international ausgewiesene Spezialisten zur Geschichte des Judentums der Einladung gefolgt. Die Vorlesungen waren ein großes mediales Ereignis; die Presse, die von Eva von Freeden mit Informationen versorgt wurde, berichtete ausführlich und positiv. Es war von vornherein geplant gewesen, die Vorlesungen zu veröffentlichen: Sie erschienen, zum Teil leicht modifiziert, $199^{2}$ unter dem Titel «Die Juden in der europäischen Geschichte» im Verlag C.H.Beck. Herausgeber war Wolfgang Beck. Die Einleitung hatte Christian Meier allein verfasst, da Thomas Nipperdey am 14. Juni $199^{2}$ verstorben war und das Ende der Reihe nicht mehr erlebt hatte. Seinem Andenken ist das Buch gewidmet.

Der Verlag organisierte großzügig die Umsetzung des Programms. Die einzelnen Vorlesungen wurden mit je 2000 DM honoriert. Reise-, Hotel- 
und gegebenenfalls Übersetzungskosten kamen hinzu. Die beiden Berater erhielten je 1500 DM. Nach den Vorlesungen fand stets ein Empfang statt, für den der Verlag aufkam. Insgesamt beliefen sich die Kosten auf über 100000 DM. ${ }^{16}$ Diese Vorlesungsreihe zum Verlagsjubiläum zu stiften, erwies sich für C.H.Beck in mehrfacher Hinsicht als eine lohnende Investition. Wie die zahlreichen Zeitungsartikel belegen, war das symbolische Kapital, das er damit erwarb, enorm: Der Verlag hatte sich in den kontroversen Diskussionen um die «Erinnerung» an die Shoah und die «Geschichtspolitik» im wiedervereinigten Deutschland zu Wort gemeldet. C.H.Beck kommunizierte der Öffentlichkeit, dass er den Zivilisationsbruch von Auschwitz ebenso zum Thema seines Programms machen werde wie die Geschichte, Religion und Kultur des europäischen Judentums - wider das Vergessen. Das Verlagsprogramm sollte jedoch nicht allein auf dem bundesrepublikanischen «Gründungsmythos Auschwitz» aufbauen und an die Opfer des Nationalsozialismus erinnern, ${ }^{17}$ sondern die jüdische Tradition breiter vergegenwärtigen. 1991 erschien auf Vorschlag der Lektorin Christine Zeile der Band «Das Schtetl. Die untergegangene Welt der osteuropäischen Juden» von Mark Zborowski und Elisabeth Herzog. Die Verlagspolitik war, wie schon im Historikerstreit, auch eine Reaktion auf die konservative Geschichtspolitik unter dem Bundeskanzler Helmut Kohl, deren Ziel es war, eine «normale` nationale Identität zu entwickeln, und die eine breite Debatte über einen systematisch intendierten Geschichtsrevisionismus auslöste. ${ }^{18}$

In dieser Situation griff C.H.Beck konsequent das breite Interesse an der jüdischen Geschichte auf und trat in eine produktive Konkurrenz mit dem «Jüdischen Verlag im Suhrkamp Verlag», dessen Programm seit 1992 erschien. Dabei gelang es dem Münchner Verlag, jüdische Historikerinnen und Historiker aus Israel und den USA als Autoren zu gewinnen und damit die rein bundesrepublikanischen Diskussionen um den Völkermord an den Juden und die deutsche Erinnerungskultur zu transzendieren. Für den Verlag nicht minder wichtig waren die Kontakte, die sich durch die Vortragsreihe zu den einzelnen Wissenschaftlern ergaben und aus denen Folgeprojekte resultierten. Es waren liberale jüdische Historiker, die C.H.Beck in Deutschland verlegte. Eine Übersetzung des konservativen Rabbiners Arthur Hertzberg etwa lehnte man ab. ${ }^{19}$ Die internationale Sichtbarkeit des Verlags wurde nicht zuletzt durch die englische Übersetzung der Vortragsreihe unter dem Titel «The Jews in European History» vergrößert, die 1994 die Hebrew Union College Press zusammen mit dem Leo Baeck Institute herausbrachte. 
Nicht dauerhaft etablieren ließ sich hingegen eine Verbindung zu Christian Meier. Der Althistoriker veröffentlichte zwar 1990 die zweite, erweiterte Auflage seiner drei Jahre zuvor im Deutschen Kunstverlag publizierten Essaysammlung «Vierzig Jahre nach Auschwitz. Deutsche Geschichtserinnerung heute» bei C.H.Beck, ließ sich aber von Ernst-Peter Wieckenberg nicht bewegen, mit althistorischen Büchern in die Wilhelmstraße zu kommen. Über einen «Römer-Essay» für die geplante Reihe «C.H. Beck Wissen» dachte er im Sommer $199^{2}$ kurz nach, nachdem Wieckenberg ihm mitgeteilt hatte, dass man «schon etwa zehn Verträge innerhalb von zwei Monaten mit sehr guten Autoren über Gegenstände der Geschichte und Kulturgeschichte» habe abschließen können. ${ }^{20}$ Im April 1993 unternahm der Cheflektor nochmals einen Anlauf, indem er Bezug nahm auf Meiers «Programm einer Geschichtsschreibung», das er im «Merkur» vorgelegt hatte: «Sehe ich das richtig, oder sind Sie derzeit der einzige, der in Deutschland sich auch Gedanken über Fragen der Vermittlung von Geschichte macht?», fragte Ernst-Peter Wieckenberg. Sein Eindruck sei gelegentlich, die Historiker glaubten, mit der etwas banalen Aufforderung, man solle wieder erzählen, habe man schon alles zu diesem Thema gesagt. Auf welchem Niveau die Ethnologie heute über ihre Schreib- und Mitteilungsformen nachdenke, das sollten sich die Historiker einmal anschauen. Umso erfreulicher finde er es, dass wenigstens Meier sich damit beschäftige, wobei für ihn auch das Konzept des Buches «Vierzig Jahre nach Auschwitz» «implizit und explizit» eine Antwort auf Fragen sei, wie man denn mit bestimmten Ereignissen der Geschichte umzugehen habe. Dann unterbreitete er dem Althistoriker ein Angebot: In seinem Aufsatz spreche Meier auch davon, dass sein Athen-Buch langsam fertig sei. «Ich bin da in einer etwas schwierigen Lage. Auf der einen Seite möchte ich nicht in den Geruch dessen kommen, der bestehende Verträge nicht respektiert. Auf der anderen Seite wissen Sie, dass unser Verlag, dass ich Ihr Buch sehr, sehr gerne hätte.» ${ }^{21}$ Christian Meier gab dem Werben nicht nach. Er hielt Wolf Jobst Siedler die Treue. Ernst-Peter Wieckenberg respektierte diese Entscheidung. Zu C.H.Beck kam Christian Meier nur noch einmal. 2002 veröffentlichte er «Von Athen bis Auschwitz. Betrachtungen zur Lage der Geschichte» im Rahmen der von C.H.Beck verlegten «KruppVorlesungen zu Politik und Geschichte am Kulturwissenschaftlichen Institut im Wissenschaftszentrum Nordrhein-Westfalen». 
Den eigentlichen Durchbruch für das neue Programm zur jüdischen Geschichte brachte die «Deutsch-jüdische Geschichte in der Neuzeit», die im Auftrag des Leo Baeck Instituts von Michael A. Meyer unter Mitwirkung von Michael Brenner 1996 und 1997 herausgegeben wurde. Das vierbändige Werk wurde gleichzeitig auch in hebräischer und englischer Sprache veröffentlicht. Damit hatte das Leo Baeck Institut das Programm, das C.H.Beck zur jüdischen Geschichte verlegte, coram publico anerkannt. Ernst-Peter Wieckenberg hatte zunächst eine ähnliche Idee im Verlag verfolgt. Er wollte eine Art Handbuch zur deutsch-jüdischen Geschichte der Neuzeit herausbringen, wurde dann aber von seinem Studienkollegen Reinhard Rürup, dem Berliner Historiker und Leiter der Gedenkstätte Topographie des Terrors, auf das Projekt des Leo Baeck Instituts hingewiesen. Als das Institut selbst Wieckenberg über das große, mit öffentlichen Mitteln geförderte Unternehmen informierte, schrieb er an Fred Grubel und regte an, das Vorhaben gemeinsam zu verwirklichen. Ein Treffen in München schloss sich an, bei dem man handelseinig wurde. Der Cheflektor nahm zudem aus dem Gespräch inhaltliche Anregungen für die weitere Entwicklung des Verlagsprogramms mit: Zum einen war nach dem Vorbild des Leo Baeck Instituts die deutsch-jüdische Geschichte nicht nur als Leidensgeschichte darzustellen, als Geschichte der Missachtung, der Verfolgung und schließlich der Vernichtung, sondern als Geschichte eines - wie immer spannungsreichen - Miteinanders und produktiven Gegeneinanders. Zum anderen wurde Wieckenberg darin bestärkt, sich nicht nur auf die deutschen Juden beschränken zu dürfen, sondern jüdische Geschichte und Kultur auch in anderen Ländern und Regionen in den Blick zu nehmen. ${ }^{22}$

Die Beweggründe des Verlags, die «Deutsch-jüdische Geschichte in der Neuzeit» zu verlegen, sprach Ernst-Peter Wieckenberg in einem Brief an Michael A. Meyer offen aus: «Vorweg schon ein Wort zu der Frage nach der Leserschaft. Wir stel-

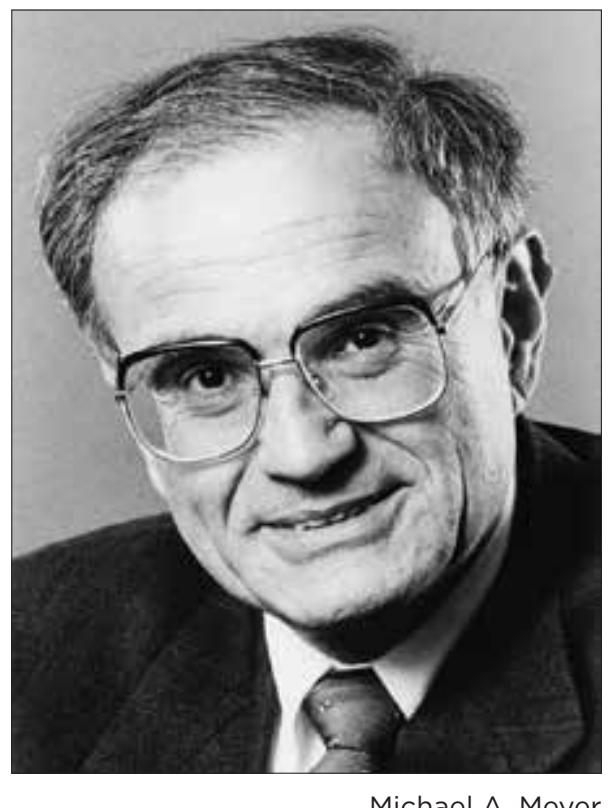

Michael A. Meyer 
len uns vor, dass das Werk für jeden Historiker von Interesse sein soll, dass es aber auch für den historisch interessierten Laien verständlich bleiben muss. Die Leserschaft wird wahrscheinlich dem Publikum entsprechen, das zu der Vorlesung erschien, zu der Sie selber einen Vortrag beigesteuert haben: Fachhistoriker, Vertreter der Nachbarwissenschaften, Studenten, gebildete Leser aus Politik und Verwaltung, allgemein historisch interessierte Laien.» ${ }^{23}$

Bereits zuvor hatte Ernst-Peter Wieckenberg in engem Kontakt mit Meyer gestanden, der auch die englische Version der Vortragsreihe herausgab, nachdem er selbst eine Übersetzung ins Gespräch gebracht hatte. ${ }^{24}$ Der Cheflektor war zudem begeistert von Meyers Buch «The Origins of the Modern Jew. Jewish Identity and European Culture in Germany, 1749-1824". Thomas Nipperdey hatte ihm, schon auf dem Krankenlager, diese Monographie zur jüdischen Geschichte ans Herz gelegt und hinzugesetzt: «Sie machen natürlich den Meyer.» ${ }^{25}$ Wieckenberg besorgte sich das Buch. Am 24. März 1992 schrieb er an den Historiker in Cincinnati, nachdem er die ersten hundert Seiten gelesen hatte: «Ich bin, um das gleich zu sagen, begeistert von der Klarheit und Differenziertheit der Darstellung und möchte versuchen, hier eine Übersetzung zu bewirken. Leicht wird es nicht sein, denn man wird eine riesige Auflage nicht wagen können, und Übersetzungen, die man nur in kleinen Auflagen herausbringt, lassen sich nur schwer kalkulieren.» Es komme als «kleines Problem» hinzu, dass es das Buch «Entstehung des modernen Judentums. Geistesgeschichte der deutschen Juden 1650-194,2» des Hamburger Judaisten Heinz Mosche Graupe gebe, das 1977 in zweiter Auflage in einem Hamburger Verlag erschienen war. ${ }^{26}$ Um Meyers Buch in deutscher Sprache veröffentlichen zu können, entschloss sich der Cheflektor, es selbst zu übersetzen. ${ }^{27}$ Wieckenberg war diese Aufgabe ein persönliches Anliegen, das er mit großer Energie verfolgte. Im Jahr 1994 erschien das Werk unter dem Titel «Von Moses Mendelssohn zu Leopold Zunz. Jüdische Identität in Deutschland 1749-1824». Wegen eines Problems des Titelschutzes konnte man nicht den Titel wählen, unter dem das Werk dann 2011 als preiswertes Paperback wieder herauskam: «Die Anfänge des modernen Judentums. Jüdische Identität in Deutschland 1749-1824"”.

Aus der professionellen Beziehung zwischen Lektor und Autor wurde eine persönliche Freundschaft. Man besprach nicht nur Buchprojekte, ${ }^{28}$ sondern auch politische Entwicklungen in Deutschland, Israel und den Vereinigten Staaten. Anfang 1993 ging Ernst-Peter Wieckenberg auf die fremdenfeindlichen Anschläge ein, die Deutschland nach der Wiederver- 
einigung erschüttert hatten: die rassistischen Ausschreitungen im sächsischen Hoyerswerda im September 1991 und in Rostock-Lichtenhagen im August 1992 sowie den rechtsextremen Brandanschlag auf eine türkische Familie im schleswig-holsteinischen Mölln am 23. November 1992. Der Cheflektor schrieb an den jüdischen Historiker und warb für eine differenzierte Betrachtung der furchtbaren Ereignisse: «Was die politischen Zustände hier angeht, so kann ich die Empörung und die Betroffenheit in Amerika und nicht nur in Amerika verstehen. Auch wir sind alles andere als glücklich. Aber ich glaube doch, dass die Situation heute anders ist als in Weimar, weil einige Bedingungen, die einen Aufstieg der Nazis in der Weimarer Republik ermöglicht haben, heute fehlen: die Industrie ist ganz und gar gegen Rassismus und Ausländerfeindlichkeit. Die Justiz und die Verwaltung sind, das wage ich zu behaupten - auch wenn es nicht-demokratische oder antidemokratische Relikte geben mag -, demokratisch gesonnen. Die Bundeswehr stellt keinen Machtfaktor dar. Das sind die Positiva. Was uns alle erschreckt hat, war das Faktum, dass es ganz offensichtlich nicht nur in dem ganz äußeren rechten Feld des gesellschaftlichen Spektrums Rechtsradikalismus und Fremdenhass gibt, dass es vielmehr diesseits dieser Felder des Spektrums Leute gibt, bei denen man eine - um es freundlich zu sagen - gewisse Labilität diagnostizieren muss. Aber gerade um die muss man sich kümmern. Und man muss ihnen zeigen, dass man ihre Haltung nicht goutiert. Das wird unsere Aufgabe in den kommenden Monaten und Jahren sein. Ich hoffe, ich bin nicht zu leichtsinnig und optimistisch, wenn ich glaube, dass wir mit diesen Problemen fertigwerden. Hässlich wird es freilich von Zeit zu Zeit dabei zugehen, und das Deutschland-Bild in der Welt, das ohnehin nicht gerade das beste ist, wird dabei weitere Kratzer abbekommen.» ${ }^{29}$ Damit hatte der Cheflektor zugleich die Aufgabe umschrieben, die der Verlag in der deutschen Zivilgesellschaft zu erfüllen hatte: mit seinem Programm aufklärerisch gegen Fremdenhass und Rechtsradikalismus, Geschichtsklitterung und Revisionismus zu wirken.

Über die «Deutsch-jüdische Geschichte in der Neuzeit» ergaben sich neue Kontakte. 1995 wurde der Historiker Michael Brenner mit dem «Beck'sche Reihe»-Band «Nach dem Holocaust. Juden in Deutschland 19451950» Autor des Verlags. Brenner hat seit 1997 den neu eingerichteten Lehrstuhl für Jüdische Geschichte und Kultur an der Ludwig-MaximiliansUniversität München inne. C.H.Beck hat durch sein Verlagsprogramm mit dazu beigetragen, dass an deutschen Hochschulen wieder intensiv über jüdische Geschichte, Kultur und Religion geforscht und gelehrt werden 
kann. Dennoch bleibt Michael Brenners Beschreibung der aktuellen Situation zutreffend: «Die Erforschung jüdischer Geschichte und Kultur ist heute in Deutschland da angekommen, wo sie sich die Wissenschaft des Judentums im 19. Jahrhundert gewünscht hätte; ihre Protagonisten, die deutschen Juden, jedoch existieren kaum noch. War die Wissenschaft des Judentums einst ein Unternehmen im Rahmen der Institutionen der jüdischen Gemeinschaft, aber außerhalb der deutschen Wissenschaft, so ist sie heute ein Projekt der deutschen Wissenschaft, aber weitgehend außerhalb des in bescheidenem Rahmen wieder aufgebauten jüdischen Lebens.» ${ }^{30}$ Michael Brenner hat weitere wichtige Bücher zur jüdischen Geschichte bei C.H.Beck veröffentlicht, darunter die Übersetzung seiner grundlegenden, an der Columbia University verfassten Dissertation über «Jüdische Kultur in der Weimarer Republik» (200o), aber auch «Propheten des Vergangenen. Jüdische Geschichtsschreibung im 19. und 20. Jahrhundert» (2006) und die «Kleine jüdische Geschichte» (2008).

Über die Vorlesungsreihe der Jahre 1991/92 konnte die Verbindung zu Saul Friedländer hergestellt werden - einem Überlebenden des Holocaust: Als Sechsjähriger war er mit seinen Eltern nach dem Einmarsch der Wehrmacht in die Tschechoslowakei aus Prag nach Frankreich geflohen. Während er selbst in einem katholischen Internat überlebte, wurden die Eltern aus Vichy-Frankreich deportiert und ermordet. Wolfgang Beck und ErnstPeter Wieckenberg waren von Friedländers Schlussvortrag tief beeindruckt und bemühten sich, ihn für ein Buchprojekt zu gewinnen. ${ }^{31}$ Der Historiker plante damals ein zweibändiges Werk über das «Dritte Reich» und die Juden, wie er Wieckenberg anlässlich seines Vortrags in München mitgeteilt hatte. Ein Vertrag mit einem amerikanischen Verlag existierte bereits. Wolfgang Beck schrieb dem Historiker im Juli 1992: «Für uns, die wir wissen, dass Sie an einem großen Werk über die Shoah arbeiten, war und ist Ihr Vortrag zugleich so etwas wie ein Vorgeschmack und auch ein Programm dessen, was Ihnen vorschwebt. Dass wir an diesem Werk verlegerisch sehr interessiert sind, wissen Sie von Herrn Wieckenberg. Ich möchte unser Interesse auch meinerseits bekräftigen.» Und einige Zeit später verstärkte der Verleger seine Bemühungen: «Peter Wieckenberg schreibt Ihnen zu Ihrem geplanten zweibändigen Werk über die Shoah, dessen deutsche Ausgabe wir auf jeden Fall verlegen möchten. Das Werk wird in unserem historischen Programm eine zentrale Stelle einnehmen. Ich hoffe sehr, dass Sie es uns anvertrauen.» ${ }^{32}$

Längere, nicht immer einfache Verhandlungen mit einer Literaturagentur, die die Rechte erworben hatte, führten zum Ziel: 1998 erschien der 
erste Band von Saul Friedländers bedeutendem Werk «Das Dritte Reich und die Juden», der «Die Jahre der Verfolgung 1933-1939» zum Gegenstand hatte. Im Herbst desselben Jahres wurde er mit dem Geschwister-SchollPreis ausgezeichnet. Jan Philipp Reemtsma formulierte in seiner Laudatio: «Saul Friedländers Buch hat das Kunststück vollbracht, beides zu sein: Erklärung lege artis und Demonstration menschlicher Freiheit und Verantwortlichkeit.» ${ }^{33}$ Während Daniel Jonah Goldhagens Buch «Hitlers willige Vollstrecker. Ganz gewöhnliche Deutsche und der Holocaust», das 1996 in deutscher Übersetzung bei Siedler erschienen war, einmal mehr die Täter inquisitorisch in den Mittelpunkt einer provozierenden Darstellung gerückt und eine heftige Kontroverse ausgelöst hatte, distanzierte sich Saul Friedländer implizit von dieser Art der Holocaustforschung und nahm konsequent die Perspektive der Opfer ein. Dabei verband er Authentizität und Analyse: Er erinnerte an die Leiden der Opfer und die Gewalt der Katastrophe und zeigte, dass der nüchterne Zugriff der historisch-kritischen Methode nicht ausreichen kann, um die Singularität der Ereignisse begreiflich zu machen. Den Quellen verlieh Friedländer eine tragende Funktion in der historischen Darstellung. Sie transportieren Unmittelbarkeit, Emotion und Empathie, mithin Eigenschaften, die seit dem Siegeszug des Historismus aus der Historiographie verbannt waren. Die historische Meistererzählung gibt dem Zeugnis der Opfer eine neue Dignität.

2006 kam der zweite und abschließende Band heraus: «Die Jahre der Vernichtung 1939-1945", den das Feuilleton und die Fachpresse in außergewöhnlicher Weise würdigten. «Wer dieses Buch gelesen hat, der wird es nicht vergessen; es ist emotional aufwühlend, intellektuell herausfordernd, es ist wahrhaftig [...] das beste Buch, das es zu diesem Thema gibt», schrieb Ulrich Herbert in der «Süddeutschen Zeitung». «Die wichtigste Darstellung des Holocaust aus der Feder eines Überlebenden und großen Gelehrten - ein Meisterwerk», urteilte Volker Ullrich in der «Zeit». Und Dieter Pohl resümierte im «Spiegel»: «Saul Friedländer hat eine exzellente Gesamtdarstellung des Holocausts geschrieben und zugleich den Opfern ein Denkmal gesetzt [...]. Wer wissen will, wie es eigentlich gewesen ist, der muss dieses Buch lesen.» ${ }^{34}$ Im November wurde der Band mit der höchsten jemals abgegebenen Stimmenzahl auf den ersten Platz der SZ/NDR-Sachbuch-Bestenliste gewählt. Dan Diner erklärte in der «Welt» den Erfolg des Werks: «Saul Friedländer schildert die Jahre der Vernichtung mit einer ungeheuren Wucht und Dramatik. Seine Erzählform erinnert an einen Filmregisseur. Und er verbindet elegant Einzelschicksale mit dem Weltgeschehen.» ${ }^{35}$ 


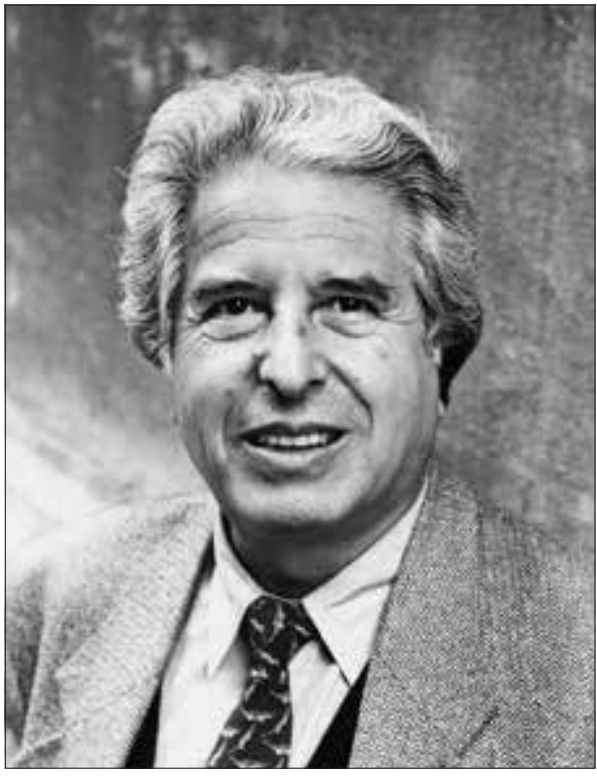

Saul Friedländer
Nachdem Friedländer im Frühjahr 2007 den Sachbuchpreis der Leipziger Buchmesse erhalten hatte, wurde er im Herbst mit dem Friedenspreis des Deutschen Buchhandels ausgezeichnet. Wolfgang Beck hatte ihn vorgeschlagen. ${ }^{36}$ Der Stiftungsrat ehrte den israelischen Historiker als «epischen Erzähler der Geschichte der Shoah», der «den Ermordeten die ihnen geraubte Würde zurückgegeben» hat. ${ }^{37}$ Ein Jahr später wurde Saul Friedländer für sein Werk auch der Pulitzer-Preis zuerkannt.

Zeitgleich mit dem ersten Band von «Das Dritte Reich und die Juden. Die Jahre der Verfolgung» veröffentlichte C.H. Beck auch eine Neuauflage von Saul Friedländers «Wenn die Erinnerung kommt» (1998); die Autobiographie war 1979 bereits in der Deutschen Verlags-Anstalt erschienen. Friedländer aber war inzwischen Beck-Autor geworden. Er fühlte und fühlt sich diesem Haus nicht nur durch das Programm verbunden, sondern auch durch die persönliche Betreuung, die ihm zuteil wurde. In der Danksagung zu dem Band «Die Jahre der Verfolgung» schrieb er: «Auf Grund meiner über dreißigjährigen Erfahrung mit Buchveröffentlichungen weiß ich die unentbehrliche Hilfe des Lektors zu schätzen. Ernst-Peter Wieckenberg gehört mit Sicherheit zu den besten, mit denen ich je zusammengearbeitet habe, und ich bin ihm für seine ständige Beratung und Umsicht außerordentlich dankbar.» ${ }^{58}$

Ernst-Peter Wieckenberg erhielt 2007 die Ehrendoktorwürde der Ludwig-Maximilians-Universität München, mit der sein Lebenswerk und sein Einsatz für die Erforschung der jüdischen Geschichte gewürdigt wurde. Über die jüdische Geschichte kam der Verlag zur Unternehmensgeschichte. Die angemessene Darstellung der Verstrickungen deutscher Firmen, Banken, Versicherungen und Konzerne in den Nationalsozialismus bedeutete dabei stets eine besondere historiographische Herausforderung. Wieckenbergs Verbindung zu dem amerikanischen Historiker Gerald D. Feldman brachte die Geschichte der «Deutschen Bank» zu C.H.Beck. ${ }^{39}$ Der von Lothar Gall, Gerald D. Feldman, Harold James, CarlLudwig Holtfrerich und Hans E. Büschgen herausgegebene Band «Die Deutsche Bank 1870-1995» von 1995 setzte durch die konsequente Abkehr 
von der Gattung der «Firmenfestschriften» und durch die wissenschaftliche Unabhängigkeit der an ihr beteiligten Historiker Maßstäbe für künftige Unternehmensgeschichten. Ein neuer Programmschwerpunkt entstand. 1997 übernahm C.H.Beck von Franz Steiner die mit zwei Heften jährlich erscheinende «Zeitschrift für Unternehmensgeschichte» sowie die die Zeitschrift begleitende Schriftenreihe. Anschlussprojekte widmeten sich u. a. der Geschichte der Deutschen Bahn, der BASF, der Swiss Re, der Konzerne Degussa, MAN, Oetker, Saint-Gobain und der Unternehmerfamilie Quandt. Ernst-Peter Wieckenberg betreute auch Feldmans Darstellung der Geschichte der Allianz und der deutschen Versicherungswirtschaft von 1933 bis 1945, die 2001 erschien. Dieser Konzern entschloss sich, an der Münchner Universität eine Gastprofessur zu stiften, die im halbjährlichen Turnus von den Islamwissenschaften und der Abteilung für Jüdische Geschichte und Kultur genutzt werden kann. Die Professoren wurden und werden von dem ehemaligen Cheflektor des Verlags C.H.Beck betreut. $^{40}$

Die jüdische Geschichte ist einer der besonders profilierten Programmbereiche der neuesten Verlagsgeschichte. Die hier veröffentlichten Bücher heben nachdrücklich die Notwendigkeit eines fortgesetzten Perspektivenwechsels ins Bewusstsein: Nicht Täter oder Opfer, sondern beide Gruppen sind in den Blick zu nehmen. Sie unterstreichen die Bedeutung einer umfassenden Betrachtung der jüdischen Geschichte in verschiedenen historischen und geographischen, kulturellen und sozialen Kontexten. Und sie lassen die Impulse erkennen, die gerade ausländische Wissenschaftler der deutschen Gesellschaft für den Umgang mit ihrer Vergangenheit gegeben haben und künftig geben können. 2012 erschien der Band «Geschichte der Juden in Deutschland von 1945 bis zur Gegenwart», der die «Deutsch-jüdische Geschichte» in vier Bänden bis zur jüngsten Gegenwart fortsetzt. Michael Brenner, Dan Diner, Nobert Frei, Lena Gorelik, Constantin Goschler, Atina Grossmann, Anthony Kauders, Tamar Lewinsky und Yfaat Weiss sind die Autoren dieses auf der Grundlage breiter Archivrecherchen geschriebenen Buches. Auch dieses Werk illustriert paradigmatisch den engen Zusammenhang von politischer Kultur und historischer Erinnerung, den das Verlagsprogramm von C.H.Beck abbildet. 
https://doi.org/10.17104/9783406654015-641, am 26.04.2023, 15:05:05

Open Access - (c) EY EY - http://www.beck-elibrary.de/agb 